\title{
Gastrointestinal Complications Post COVID-19 Infection Can be Quite Challenging and Variable in Nature
}

\section{Omar S Mansour*}

Consultant Colorectal and Laparoscopic General Surgeon, Assistant Professor and

Clinical Lecturer in General Surgery, Department of Surgery, School of Medicine, Al

Balqa Applied University, Al Salt, Jordan

*Corresponding Author: Omar S Mansour, Consultant Colorectal and Laparoscopic

General Surgeon, Assistant Professor and Clinical Lecturer in General Surgery,

Department of Surgery, School of Medicine, Al Balqa Applied University, Al Salt,

Jordan.

DOI: 10.31080/ASGIS.2021.04.0260

Keywords: COVID-19; Complications; GORD; Anal Fissure; Constipation

Since the breakout of COVID-19 infection, our surgical practice had changed dramatically due to the fast spread of the SARS-COV-2 virus [1]. Although COVID-19 infection is usually a respiratory disease, it was reported before that it can present as DIC (disseminated intravascular coagulation) especially in life threatening infections [2]. It has also been reported that gastrointestinal symptoms can present in around $80 \%$ of hospitalized patients with severe COVID-19 infections [3]. In my colorectal clinic, I reviewed few COVID-19 patients suffering from co-existing or post COVID-19 gastrointestinal complications. Few had exacerbating of already diagnosed conditions, while others had new onset diseases and symptoms. Most of these patients had a variety of different GI complications that lasted for more than 6 weeks post COVID-19 infection diagnosis confirmed with PCR testing.

The first patient was a 46-year-old male who presented with severe bloating, generalized abdominal pain and constipation. These symptoms started immediately post COVID-19 infection. He also presented to the emergency department with severe epigastric and lower chest pain. An emergency CT scan of the abdomen and pelvis failed to show any urgent GI pathology. A double endoscopy on the following day showed severe duodenitis in D1 and evidence of recent constipation. $H$. pylori rapid urease test was positive. Random colonic and TI biopsies ruled out colitis and enteritis. A polyp was excised from the descending colon and histol-
Received: April 28, 2021

Published: May 01, 2021

(C) All rights are reserved by $\mathbf{0 m a r} \mathbf{S}$

Mansour. ogy exam confirmed it to be a benign leiomyoma. The patient was still in remission two weeks post endoscopy.

The second patient was 25-year-old female who was well known to the clinic with recurrent GORD secondary to a moderate sized hiatus hernia. She acutely presented with severe vomiting and epigastric pain post COVID-19 infection. I performed a gastroscopy as an emergency and the hiatus hernia was much bigger than what was seen in the previous endoscopy. The hiatus hernia was confirmed by a barium swallow exam. High dose PPIs and antiemetics were started and the patient improved dramatically.

The third patient was a male patient who presented with severe diarrhea, anal pain and intermittent rectal bleeding. These were all new symptoms. Clinical examination and proctoscopy showed new fourth degree haemorrhoids and an anal fissure. He opted for conservative treatment with GTN cream, high fibre diet and laxatives. He quite improved on this treatment.

The fourth patient, a 66-year-old female who presented with GORD, dyspepsia and early satiety. She declined endoscopy but improved with conservative management including, oral prednisolone, PPIs and analgesia. A sixth male patient presented with severe bloating and abdominal pain more than 3 months post infection. He opted for conservative treatment. Another 48-year-old female pre- 
sented with severe generalized abdominal pain, severe diarrhoea and inability to tolerate oral intake. She was treated conservatively with intravenous PPIs, IV fluids and analgesia. Her liver function enzymes were deranged (not uncommon in SARS-COV-2 infection). The severe GI symptoms lasted for one week. She is currently still in remission one-month post cure.

In conclusion, gastrointestinal complications secondary to COVID-19 infection can present either early or late during the COVID-19 infection or even after cure from this disease. These GI complications can present either as exacerbating of chronic diseases or as new onset pathologies. These conditions should be approached carefully and investigated thoroughly. Almost all of these GI complications can be treated successfully with conservative measures and as per current GI guidelines. If the GI guidelines are not followed carefully, many misdiagnoses would result, which can lead to patient's suffering [4].

\section{Bibliography}

1. Omar S Mansour. "Why Surgeons Should Continuously Revise their Basic Medical Knowledge and be Always Medically Updated". Acta Scientific Gastrointestinal Disorders 4.2 (2021): $1-2$.

2. Omar S Mansour. "Could a Cascade of Overwhelming Disseminated Intravascular Coagulation (DIC) be the Primary Pathology in Life Threatening COVID-19 Infections?" Acta Scientific Gastrointestinal Disorders 3.6 (2020): 06-11.

3. Haytham MA Kaafaranim., et al. "Gastrointestinal Complications in Critically Ill Patients With COVID-19". Annals of Surgery 272.2 (2020): e61-e62.

4. Omar S Mansour. "Elevated Fecal Calprotectin Levels Can be Misleading if Guidelines are Not Properly Followed". Acta Scientific Gastrointestinal Disorders 4.3 (2021): 46-47.

Volume 4 Issue 6 June 2021

(C) All rights are reserved by Omar S Mansour. 